\title{
The 2019 novel cornoavirus pneumonia with onset of oculomotor nerve palsy: a case study
}

\author{
Heng Wei ${ }^{1} \mathbb{D} \cdot$ Hongxiang Yin ${ }^{1} \cdot$ Min Huang $^{2} \cdot$ Zhenli Guo $^{1}$
}

Received: 19 February 2020 / Accepted: 20 February 2020 / Published online: 25 February 2020

C) Springer-Verlag GmbH Germany, part of Springer Nature 2020

\section{Dear Sirs,}

On December 31, 2019, several cases of pneumonia of unknown etiology have been reported in Wuhan, Hubei province, China [1-3]. On January 7, 2020, Chinese health authorities confirmed that these cases were associated with a novel coronavirus, which was subsequently named 2019nCoV by WHO [4]. Previous study [5] reported that virus infection can cause several neurological complications, including polyneuritis, Guillain-Barre syndrome (GBS), meningitis, encephalomyelitis, and encephalopathy. We describe a rare case of 2019-CoV infection and acute unilateral isolated oculomotor nerve palsy. In this case, the diagnosis was made based on the chest computed CT manifestations and throat swab sample test.

A 62-year-old man was admitted to our department with a 5-day history of persistent diplopia and a droopy left eyelid. During initial hospital assessment, he endorsed limb weakness and poor spirit. He denied any fever, neck stiffness, headache, cough, shortness of breath, chest pain, or photophobia. He had a history of alcohol and tobacco use, type II diabetes mellitus and hypertension (both well controlled by drugs), and lacunar infarction (without sequela).

On examination, it revealed body temperature of $36.5^{\circ} \mathrm{C}$ $\left(97.7^{\circ} \mathrm{F}\right)$, blood pressure of $142 / 72 \mathrm{mmHg}$, respiratory rate of 22 breaths per minute, pulse rate of 70 beats per minute and oxygen saturation of $95 \%$ while the patient was breathing ambient air. There were coarse rales in the both lung field. The patient was alert and oriented to person, place, and

Heng Wei

tonywei2011@126.com

1 Department of Neurology, Hubei Provincial Hospital of Integrated Chinese and Western Medicine, Hubei University of Chinese Medicine, Wuhan 430000, China

2 Department of Endocrinology, Hubei Provincial Hospital of Integrated Chinese and Western Medicine, Hubei University of Chinese Medicine, Wuhan 430000, China time. His speech was fluent. Pupils were $3 \mathrm{~mm}$ and equally reactive to light. He had complete ptosis of the left eyelid, and his left eye was down and out at rest. The left eye was unable to adduct and look up. Left eyelid closure was weak. Both eyes were without orbital pain. Hearing was intact. No palate or tongue weakness/asymmetry was noted. Strength in upper and lower limbs was 5/5 throughout. Deep tendon reflexes were $2+$ and symmetric throughout. Toes were downgoing bilaterally. Sensation to light touch, pinprick, and temperature was intact on the two sides. No pathological reflection of Babinski's sign is induced. Romberg test was negative when eyes were open or close. The sign of meningeal irritation was negative. The laboratory results showed white blood cell count: $9.45 \times 10^{9} / \mathrm{L}$ and neutrophil percentage: $69.6 \%$ were normal. Random blood glucose was $9.2 \mathrm{mmol} / \mathrm{L}$ (normal range $0-11.1 \mathrm{mmol} / \mathrm{L}$ ) and hemoglobin A1C was $6.1 \%$ (normal range 0-6.2\%). Respiratory pathogens test showed influenza $\mathrm{A}$ and $\mathrm{B}$ virus antigen, mycoplasma pneumonia antigen, adenovirus antigen, and syncytial virus were all negative. However, inflammatory markers were significantly elevated C-reactive protein $(142.21 \mathrm{mg} / \mathrm{L}$; normal range $0-10 \mathrm{mg} / \mathrm{L}$ ) and Serum amyloid A protein (300.00 mg/L; normal range $0-10 \mathrm{mg} / \mathrm{L})$. Erythrocyte sedimentation rate was elevated $(91.6 \mathrm{~mm} / \mathrm{h}$; normal range 0-15 mm/h). Magnetic resonance imaging (MRI) was not found new infarction, bleeding of brainstem or pituitary apoplexy, tumor, and multiple sclerosis (Figs. 1, 2). Magnetic resonance angiography (MRA) was not found aneurysms (Fig. 3). Unenhanced chest CT showed wide, multiple, diffuse ground-glass opacities in both lungs (Figs. 4, 5).On hospital day 2 , his illness evolved progressively with presenting fever and dyspnea, and body temperature was elevated to $38.9^{\circ} \mathrm{C}\left(102{ }^{\circ} \mathrm{F}\right)$. Oxygen saturation was declined to $90 \%$ while the patient was breathing ambient air. Hence, oxygen therapy via nasal catheter was given to the patient, which merely maintained oxygen saturation of 93-95\%. Meanwhile, the son of patient presented cough and limb weakness, so the chest CT was done and found the multiple 
Fig. 1 MRI FLAIR reveals normal

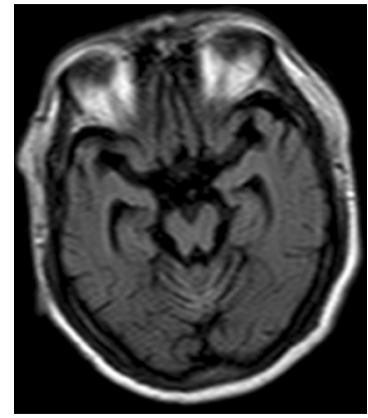

Fig. 2 Sagittal MRI T1 reveals normal

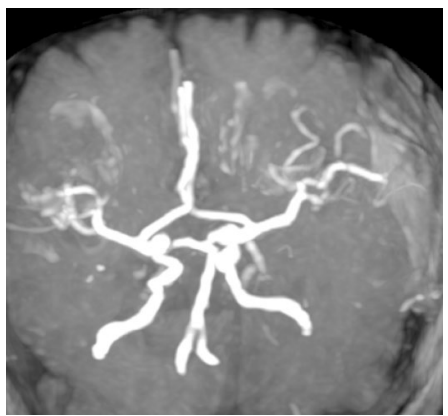

Fig. 3 MRA reveals no intracranial artery stenosis and no aneurysms

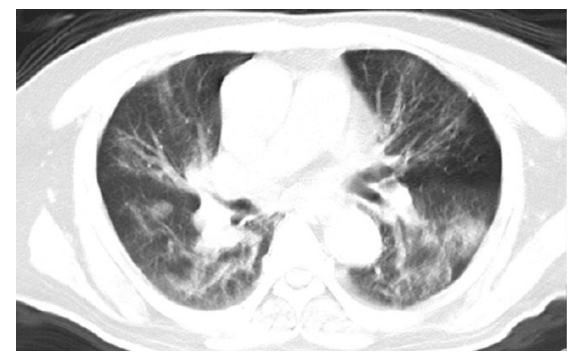

Fig. 4 Chest CT shows multiple, sporadic peripheral ground-glass opacities in both lungs

ground-glass opacities in both lungs (Fig. 6) like his father's CT. Due to the first onset of uncommon infected symptom,

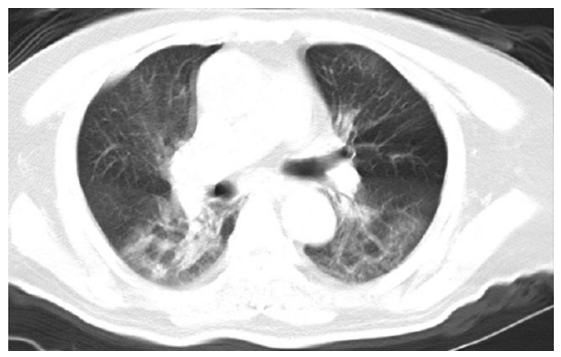

Fig. 5 Chest CT shows multiple, sporadic peripheral ground-glass opacities in both lungs

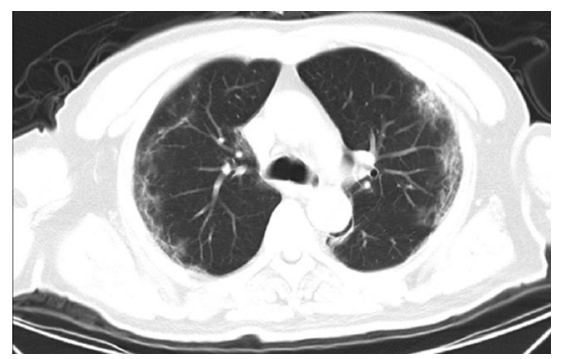

Fig. 6 Chest CT shows peripheral ground-glass opacities in both lungs

serious $\mathrm{CT}$ imaging and family aggregation, the contagious disease became possible. Subsequently, an outbreak of novel coronavirus pneumonia was in Wuhan, Hubei province, China and spreaded rapidly, where the patient lived and worked in. The patient's throat swab sample was positive for the 2019-nCoV by Chinese Center for Disease Control and Prevention (CDC). Finally, according to epidemiologic characteristics, clinical features, laboratory findings, chest images, family aggregation, throat swab sample test, and the diagnosis of 2019-nCoV infected pneumonia were made.

The onset of oculomotor nerve palsy was associated with several disorders, including intracranial aneurysm, cerebrovascular disease, brain tumor, demyelination disease, peripheral neuropathy, inflammatory disease, and so on. Intracranial aneurysm is the leading cause of oculomotor nerve palsy. The main clinical manifestations include headache, ptosis, and restriction in eye movements with or without changes in the pupil. The MRA of patient was negative and the clinical manifestations were inconformity for aneurysm. Cerebrovascular disease involved infarction or bleeding of brainstem, pituitary apoplexy was excluded by MRI scan. Short course of disease and MRI scan also excluded brain tumor. Owing to the age of patient and MRI scan, the demyelination disease was not taken into account. The diabetes mellitus was well controlled with random blood glucose and hemoglobin $\mathrm{A} 1 \mathrm{C}$ at normal range. Therefore, diabetic peripheral neuropathy was excluded. Some patients of GBS 
were associated with oculomotor nerve palsy. Nonetheless, the patient's tendon reflexes and sensation test were normal which were not in accord with GBS. Finally, infected disease was in accord with the patient. Patient's chest CT manifestation and throat swab sample were positive for the 2019nCoV. The 2019-nCoV pneumonia was likely to interpret that the chest CT manifestations and inflammatory reaction resulted in oculomotor nerve palsy.

From the patient admission, the patient received moxifloxacin (400 mg once every day), oseltamivir ( $75 \mathrm{mg}$ once every day), methylprednisolone (40 mg once every day), ribavirin ( $0.5 \mathrm{~g}$ once every day), lopinavir ( $400 \mathrm{mg}$ twice every day), and immunoglobulin $(0.4 \mathrm{~g} / \mathrm{kg}$ once every day) combined treatments. During this time, oxygen therapy was persistent supportive treatment. Antipyretic therapy consisting of $650 \mathrm{mg}$ of acetaminophen every $8 \mathrm{~h}$ and $600 \mathrm{mg}$ of ibuprofen every $12 \mathrm{~h}$. Unfortunately, his illness got worse and he was dead at admission day 12 because of respiratory failure.

In December 2019, a large number of infected pneumonia cases suddenly appeared in Wuhan. The etiology of these infections was the 2019-nCoV, possibly connected to zoonotic or environmental exposure from the Huanan seafood market in Wuhan. Human coronavirus is one of the main pathogens of respiratory infection. The two highly pathogenic viruses, SARSCoV and MERSCoV, cause severe respiratory syndrome in humans and four other human corona viruses (HCoV229E, HCoVOC43, HCoVHKU1, and HCoVNL63) induce mild upper respiratory disease [6]. The sequence of $2019 \mathrm{nCoV}$ is relatively different from the six other coronavirus subtypes but can be classified as betacoronavirus. The 2019-nCoV uses the receptor, ACE2 (angiotensin converting enzyme 2) to infect cells [7]. The process of infection generates high levels of angiotensin II (Ang II), which can lead to increases in vascular permeability and pulmonary oedema, even often lethal lung failure in patients [8]. Owing to 2019-nCoV infections, high amount of cytokines and corticosteroids was used frequently for treatment of patients with severe illness, for possible benefit by reducing inflammatory-induced lung injury. Some studies reported that receiving corticosteroids treatment delayed viral clearance [9-11]. Therefore, according to WHO interim guidance, corticosteroids should not be routinely given systemically [12]. According to the current data, the $2019 \mathrm{nCoV}$ is a highly contagious virus with mortality $11-15 \%[3,5]$. This report describes a case involving an adult patient first onset of acute left oculomotor nerve palsy with 2019-nCoV infected pneumonia that was identified based on chest CT findings and throat swab sample test. In this case, no clinical, laboratory, or imaging examinations showed any indication of an underlying structural cause of the oculomotor nerve injury, suggesting that the 2019-nCoV infection might have caused the acute oculomotor nerve palsy in this case. Why focal neurological deficits may arise following 2019-nCoV infections remains unclear. Pathological mechanisms, such as virus infection invasion or inflammatory factors accompanying the effects, are commonly suggested. On the other hand, the virus infection may directly damage the myelin sheaths and surrounding axons due to complications such as GBS, acute disseminated encephalomyelitis, transverse myelitis, and optic neuritis. The hypothesis suggests that oculomotor nerve palsy could be triggered by the same mechanism. The 2019-nCov-induced waterfall inflammation reaction led to respiratory failure and resulted in death. Unfortunately, cerebrospinal fluid examination was not examined. Although the MRI test was negative, whether the inflammatory factors invaded brainstem was unknown. To the best of my knowledge, the case was the first reported that the 2019-nCoV infection resulted in oculomotor nerve palsy. It is possible that the same symptoms or signs may be observed with rare etiology.

Funding This work received a grant from the State Administration of Traditional Chinese Medicine project of clinical cooperation between Chinese and Western medicine for major difficult diseases, China (Grant number SATCM-20180339).

\section{Compliance with ethical standards}

Conflicts of interest The authors declare that there are no competing interests associated with the manuscript.

Ethical approval This study has been approved by an appropriate ethics committee and has been performed in accordance with the ethical standards laid down in the 1964 Declaration of Helsinki and its later amendments.

Informed consent Written informed consent was obtained from the son of patient for publication of this case report and any accompanying images.

\section{References}

1. Lu H, Stratton CW, Tang YW (2020) Outbreak of pneumonia of unknown etiology in Wuhan China: the mystery and the miracle. J Med Virol. https://doi.org/10.1002/jmv.25678

2. Hui DS, Azhar IE, Madani TA et al (2020) The continuing 2019 $\mathrm{nCoV}$ epidemic threat of novel coronaviruses to global health the latest 2019 novel coronavirus outbreak in Wuhan China. Int J Infect Dis 91(264):266

3. Huang C, Wang Y, Li X et al (2020) Clinical features of patients infected with 2019 novel coronavirus in Wuhan China. Lancet. https://doi.org/10.1016/S0140-6736(20)30183-5

4. World Health Organization. Novel coronavirus(2019-nCoV) situation report-8. https://www.who.int/docs/default-source/coronaviru se/situation-reports/20200128-sitrep-8-ncov-cleared.pdf?sfvrs $\mathrm{n}=8 \mathrm{~b} 671 \mathrm{ce} 5 \_2$. Accessed 28 Jan 2020

5. Studahl M (2003) Influenza virus and CNS manifestations. J Clin Virol 28:225-232

6. Chen NS, Zhou M, Dong X et al (2020) Epidemiological and clinical characteristics of 99 cases of 2019 novel coronavirus 
pneumonia in Wuhan, China: a descriptive study. Lancet. https:// doi.org/10.1016/S0140-6736(20)30211-7

7. Zhou P, Yang XL, Wang XG et al (2020) A pneumonia outbreak associated with a new coronavirus of probable bat origin. Nature. https://doi.org/10.1038/s41586-020-2012-7

8. Fyhrquist F, Saijonmaa O (2008) Renin-angiotensin system revisited. J Intern Med 264:224-236

9. Stockman LJ, Bellamy R, Garner P (2006) SARS: systematic review of treatment effects. PLoS Med 3:e343

10. Lansbury L, Rodrigo C, Leonardi-Bee J et al (2019) Corticosteroids as adjunctive therapy in the treatment of influenza. Cochrane Database Syst Rev 2:CD010406
11. Arabi YM, Mandourah Y, Al-Hameed F et al (2018) Corticosteroid therapy for critically ill patients with Middle East respiratory syndrome. Am J Respir Crit Care Med 197:757-767

12. WHO (2020) Clinical management of severe acute respiratory infection when novel coronavirus (nCoV) infection is suspected. https://www.who.int/internal-publications-detail/clinicalmanagem ent-of-severe-acute-respiratory-infection-when-novelcoronaviru s-(ncov)-infection-is-suspected. Accessed 19 Jan 2020. 